\title{
BMJ Open Finnish study of intraoperative irrigation versus drain alone after evacuation of chronic subdural haematoma (FINISH): a study protocol for a multicentre randomised controlled trial
}

Pihla Tommiska, ${ }^{1}$ Rahul Raj (1) , ${ }^{1}$ Christoph Schwartz, ${ }^{1,2}$ Riku Kivisaari, ${ }^{1}$ T Luostarinen, ${ }^{3}$ Jarno Satopää, ${ }^{1}$ Simo Taimela (D) , ${ }^{4,5}$ Teppo Järvinen (D) , 4,5 Jonas Ranstam, ${ }^{6}$ Janek Frantzen, ${ }^{7}$ Jussi Posti, ${ }^{7}$ Teemu M Luoto, ${ }^{8}$ Ville Leinonen, ${ }^{9}$ Sami Tetri, ${ }^{10}$ Timo Koivisto, ${ }^{9}$ Kimmo Lönnrot (D) ${ }^{1}$

To cite: Tommiska P, Raj R, Schwartz C, et al. Finnish study of intraoperative irrigation versus drain alone after evacuation of chronic subdural haematoma (FINISH): a study protocol for a multicentre randomised controlled trial. BMJ Open 2020;10:e038275. doi:10.1136/ bmjopen-2020-038275

- Prepublication history and additional material for this paper are available online. To view these files, please visit the journal online (http://dx.doi. org/10.1136/bmjopen-2020038275).

PT and RR contributed equally.

Received 05 March 2020

Revised 13 May 2020

Accepted 19 May 2020

Check for updates

(C) Author(s) (or their employer(s)) 2020. Re-use permitted under CC BY-NC. No commercial re-use. See rights and permissions. Published by BMJ.

For numbered affiliations see end of article.

Correspondence to

Dr Rahul Raj;

rahul.raj@helsinki.fi

\section{ABSTRACT}

Introduction Chronic subdural haematomas (CSDHs) are one of the most common neurosurgical conditions. The goal of surgery is to alleviate symptoms and minimise the risk of symptomatic recurrences. In the past, reoperation rates as high as $20 \%-30 \%$ were described for $\mathrm{CSDH}$ recurrences. However, following the introduction of subdural drainage, reoperation rates dropped to approximately $10 \%$. The standard surgical technique includes burr-hole craniostomy, followed by intraoperative irrigation and placement of subdural drainage. Yet, the role of intraoperative irrigation has not been established. If there is no difference in recurrence rates between intraoperative irrigation and no irrigation, CSDH surgery could be carried out faster and more safely by omitting the step of irrigation. The aim of this multicentre randomised controlled trial is to study whether no intraoperative irrigation and subdural drainage results in non-inferior outcome compared with intraoperative irrigation and subdural drainage following burr-hole craniostomy of CSDH.

Methods and analysis This is a prospective, randomised, controlled, parallel group, non-inferiority multicentre trial comparing single burr-hole evacuation of CSDH with intraoperative irrigation and evacuation of CSDH without irrigation. In both groups, a passive subdural drain is used for 48 hours as a standard of treatment. The primary outcome is symptomatic $\mathrm{CSDH}$ recurrence requiring reoperation within 6 months. The predefined non-inferiority margin for the primary outcome is $7.5 \%$. To achieve a $2.5 \%$ level of significance and $80 \%$ power, we will randomise 270 patients per group. Secondary outcomes include modified Rankin Scale, rate of mortality, duration of operation, length of hospital stay, adverse events and change in volume of CSDH.

Ethics and dissemination The study was approved by the institutional review board of the Helsinki and Uusimaa Hospital District (HUS/3035/2019 §238) and duly registered at ClinicalTrials.gov. We will disseminate the
Strengths and limitations of this study

This is a multicentre nationwide prospective randomised controlled trial, with a pragmatic trial design to increase generalisability.

- The study was designed in collaboration with patient organisation experts.

- The healthcare system in Finland facilitates the follow-up of patients (particularly with respect to our primary outcome, symptomatic chronic subdural haematoma (CSDH) requiring reoperation) as $\mathrm{CSDH}$ surgery is centralised to the five neurosurgical departments participating in the trial.

- Although the surgeon performing the surgery obviously cannot be blinded to the group assignment, we have tried to maintain the masking of the treatment allocation by not disclosing it in the healthcare records.

findings of this study through peer-reviewed publications and conference presentations.

Trial registration number NCT04203550

\section{INTRODUCTION}

Chronic subdural haematoma (CSDH) is the most common type of intracranial haemorrhage and one of the most common clinical diagnoses necessitating neurosurgical treatment. CSDHs are typically caused by minor head trauma and consecutive tearing of bridging veins, leading to a haemorrhage in between the dura mater and the arachnoid membrane. The delay in the actual diagnosis of CSDH can be quite substantial due to the difficulty of the diagnosis in the early phase when neurological symptoms-such 
as progressive headache, mental deterioration or confusion, or deterioration of the patient's overall health-are quite unspecific. However, when the disease progresses and causes more direct compression to the underlying brain tissue, more specific progressive neurological signs ensue, including motor and sensory deficits, dysphasia and epileptic seizures, and the diagnosis becomes more evident. If left untreated, CSDH may also lead to loss of consciousness or even death. The definite diagnosis of CSDH is most commonly based on CT or MRI of the brain. On a CT scan, CSDH is usually of hypodense or isodense character and will feature a concavo-convex shape between the skull and the cortex.

For symptomatic CSDHs, the treatment is operative. The mainstay of treatment includes burr-hole craniostomy and intraoperative intracranial irrigation, followed by subdural drainage. ${ }^{1}$ With current treatment strategies, the recurrence rate after CSDH treatment is approximately $10 \% .^{2}$ Low risk of bias evidence exists on the role of subdural drain in recurrence rate reduction but the role of intraoperative irrigation is more controversial. Our literature review revealed a total of 10 studies assessing the effect of intraoperative irrigation: only one study employed a randomised study protocol ${ }^{3}$ while the others were retrospective analyses. Sample sizes ranged from 56 to 186 patients, and the most commonly used outcome was the rate of haematoma recurrence. Of these 10 studies, two studies found that intraoperative irrigation was associated with a significantly lower recurrence rate in comparison to no intraoperative irrigation, ${ }^{45}$ six studies found no difference in recurrence rates between intraoperative and no intraoperative irrigation, ${ }^{3}{ }^{6-10}$ and two studies found that no intraoperative irrigation was associated with a significantly lower recurrence rate compared with irrigation. ${ }^{11} 12$

It is possible that intraoperative irrigation is an unnecessary prolongation of the surgical procedure, thereby increasing the risk for infections, rebleeding and the stress levels of patients undergoing the procedure under local anaesthesia. There is also evidence to suggests that irrigation per se may be harmful. There are reports of increased risk of treatment-associated morbidity and complications such as postoperative pneumocephalus ${ }^{911} 13$ and also of direct irrigation-induced intracerebral and subarachnoid haemorrhage. ${ }^{14}$

We designed a pragmatic, parallel group, randomised, controlled multicentre non-inferiority trial to compare the use of intraoperative irrigation with no intraoperative irrigation for the operation of symptomatic CSDH (by burr-hole craniostomy and subdural drainage for 48 hours). We hypothesise that a treatment that involves no intraoperative irrigation results in non-inferior outcome compared with a treatment that involves intraoperative irrigation. Non-inferiority of the new treatment (no irrigation) with respect to the gold-standard treatment (irrigation) is of interest on the premise that the new treatment has some other advantages, such as shorter operative time and therefore reduced stress to patient, reduced cost, fewer adverse events (AEs) and technically more simple. ${ }^{15}$ We consider non-inferiority proven if the rate of recurrence in the no-irrigation group (N-IR) is within the predefined non-inferiority margin of the rate observed in the IR together with no significantly increased risk of harm.

\section{MATERIALS AND ANALYSIS}

\section{Overview of study design}

Finnish study of intraoperative irrigation versus drain alone after evacuation of chronic subdural haematoma (FINISH) is a multicentre, prospective non-inferiority randomised controlled trial, with the primary objective to compare intraoperative irrigation to no irrigation in the treatment of CSDH by single burr-hole craniostomy and subdural drainage. Except for randomisation to irrigation versus no irrigation, the management of study participants will not differ. Eligible participants are block randomised in a 1:1 allocation rate to one of two arms: (1) intraoperative irrigation or (2) no intraoperative irrigation.

This protocol has been written according to the Standard Protocols Items: Recommendations for Interventional Trials (SPIRIT) guidelines for reporting a randomised controlled trial study protocol (the SPIRIT Figure and Checklist are available as online supplementary additional file 1). ${ }^{16}$ A summary of the trial is shown in online supplementary additional file 2 .

\section{Study settings}

Participating sites are the neurosurgical departments at Helsinki University Hospital (Helsinki, Finland), Kuopio University Hospital (Kuopio, Finland), Tampere University Hospital (Tampere, Finland), Turku University Hospital (Turku, Finland) and Oulu University Hospital (Oulu, Finland). All these five units are tertiary referral centres and the only units delivering neurosurgical care in Finland.

\section{Participant selection and recruiting process}

We will screen all patients who are referred for CSDH surgery to the aforementioned departments of neurosurgery for trial eligibility. A standard clinical examination and a brain CT or MRI examination will be performed. Patients with clinical and imaging findings consistent with a diagnosis of symptomatic CSDH and considered to benefit from operative treatment of CSDH by single burrhole evacuation will be asked to participate in the trial.

\section{Inclusion criteria}

- Patients with a symptomatic unilateral or bilateral CSDH requiring burr-hole evacuation.

- Predominantly hypodense or isodense on CT imaging (or chronic hematoma on MRI).

- Clinical symptoms correlating with the CSDH.

- Patients with bilaterally operated CSDHs will be treated with the same protocol on both sides and analysed as a single study participant. 
- Patients older than 18 years of age.

\section{Exclusion criteria}

- CSDH requiring surgical treatment other than burrhole evacuation (eg, craniotomy).

- CSDH in a patient who has a cerebrospinal fluid shunt.

- Patients who have undergone any prior intracranial surgery.

- Comatose patients (Glasgow Coma Scale (GCS) score eight or lower) with absent motor responses to painful stimuli; decerebrate or decorticate posturing), where rapid haematoma evacuation is required.

- Patient's postoperative cooperation is suspected to be insufficient for drain usage (ie, disoriented or semiconscious patient).

- Patient who has received active treatment for a haematogenic malignancy within the previous 5 years.

- Patient with a central nervous system malignancy or tumour that may cause the patient's current symptoms or may interfere with the operation. For example, a small incidental meningioma without associated brain oedema, not in the vicinity of the planned burr hole, is not an exclusion criterion.

- Patient has an acute infection that requires antibiotic treatment.

- Patient has a high risk of life-threatening thrombosis (eg, recent coronary stent, intracranial stent, recent pulmonary embolism, low pressure cardiac valve replacement (mitral or tricuspid valve replacement)) and discontinuation of antithrombotic medication is not recommended.

\section{Informed consent}

At the first appointment in the emergency department or the neurosurgical ward, the attending neurosurgeon will provide the patients with detailed written and oral information on the trial and ask patients to sign an informed consent form. Withdrawal from the study is possible at any time, without affecting the course of conventional treatment, in accordance with the latest version of the Declaration of Helsinki 2013. ${ }^{17}$

Due to the nature and emergency aspects of the disease (mass effect on the brain causing confusion and disorientation, lowered level of consciousness requiring urgent surgery), some patients will not be able to give written consent prior to randomisation. If the patient is unable to give written consent prior to the randomisation, delayed consent will be sought. In these cases, oral consent will be obtained from the next of kin after providing information regarding the trial. Following oral consent from the next of kin, the patient can be randomised. Following randomisation and surgery, written consent will primarily be obtained from the patient. However, in case of the patient being unable to give written consent due to neurological disability, written consent is obtained from the next of kin. In these cases, the next of kin has the right to withdraw the patient's consent at any time. Patients who are eligible for the trial but are not willing to undergo randomisation will be asked to be included in a simultaneous, pragmatic follow-up cohort.

Participants will be asked to sign the local Biobank agreements in order to collect and store subdural fluid samples and two venous blood samples $(2 \times 10 \mathrm{~mL})$.

\section{Collected data}

We will document data in the electronic case report form (eCRF) preoperatively, intraoperatively and within $48-72$ hours postoperatively, as well as at 6 weeks $( \pm 2$ weeks) and at 6 months (see table 1 for table of events). All patients' preoperative and postoperative head CT or MR images will be sent to the Picture Archiving and Communication System of the methods centre (Helsinki University Hospital) for analysis. Ten per cent of all images will be double read by independent assessors blinded to

\begin{tabular}{|c|c|c|c|c|c|}
\hline Assessment & Baseline & Surgery & 48-72hours & 6 weeks & 6 months \\
\hline Informed consent & $x$ & & & & \\
\hline Randomisation & & $x$ & & & \\
\hline Demographics & $x$ & & & & \\
\hline Antithrombotic medication & $x$ & & $x$ & $x$ & $x$ \\
\hline Neurological symptoms & $x$ & & $x$ & $x$ & $x$ \\
\hline Residence and mobility & $\mathrm{x}$ & & & $\mathrm{x}$ & $x$ \\
\hline Imaging & $x$ & & & $x$ & \\
\hline Surgical details & & $x$ & & & \\
\hline Modified Rankin Scale & $x$ & & $x$ & $x$ & $x$ \\
\hline Survival status & & & & & $x$ \\
\hline Need for ipsilateral CSDH reoperation & & & & & $x$ \\
\hline Adverse events & & & $\mathrm{x}$ & $\mathrm{x}$ & $\mathrm{X}$ \\
\hline
\end{tabular}

$\mathrm{CSDH}$, chronic subdural haematoma. 
other patient information. To preserve confidentiality, all participants are allocated a unique study identifier during the recruitment process, which is used on all data collection forms. All study documentation is held in secure offices, and the study researchers operate according to a signed code of confidentiality. All data are entered into a password-secured database by the data managers.

\section{Surgical technique}

Current management of CSDHs at all participating centres includes single burr-hole evacuation with intraoperative irrigation followed by passive subdural drainage. As a routine, all burr-hole craniostomies are performed under local anaesthesia, often combined with intravenous sedation with benzodiazepines and/ or opioids during the operation. General anaesthesia is only used if the neurosurgeon or the anaesthesiologist considers it unsafe to perform the procedure under local anaesthesia. Routine preoperative antibiotic is given according to local protocols (normally a secondgeneration cephalosporin 30-60 min prior to incision). Typically, the surgeon drills one $14 \mathrm{~mm}$ burr hole over the maximum convexity of the CSDH. In case of bilateral CSDHs, the surgeon performs the same procedure on both sides. If irrigation is used, after opening the dura, the surgeon irrigates the subdural collection with warm (body temperature) Ringer's lactate saline until rinsing appears clear or at least $200 \mathrm{~mL}$ (in case of bilateral CSHD, $200 \mathrm{~mL}$ per side, ie, $400 \mathrm{~mL}$ total). After that, the surgeon will insert the subdural drain $3-5 \mathrm{~cm}$ deep and parallel to skull. The position of the drain (anterior, posterior) is left to the discretion of the physician. Burr hole covers or haemostatics are not routinely used (eg, Spongostan, Tachosil). The type of subdural drain is not standardised, but all study centres use $10 \mathrm{~F}$ drains. Following drain insertion, the distal end is tunnelled approximately $4-5 \mathrm{~cm}$ from the incision and connected to a passive ventricular drainage bag (through a non-return valve) and the skin incision is closed in two layers (normally absorbable 3-0 suture for subcutis/galea and non-absorbable 4-0 suture for skin). The drain is fixed to the skin in a secure way. The drainto-skin fixation technique is left to the discretion of the operating surgeon. The drainage bag is positioned at bed level. The duration of subdural drainage is 48 hours ( \pm 12 hours). ${ }^{18} 19$ Patient mobilisation is allowed during drainage (drain is kept open). Prophylactic antibiotics during drainage are not routinely used.

\section{Randomisation}

Patients will be randomised in a 1:1 allocation ratio stratified only by study centre. We will use a random block randomisation technique, with a random block size of 4, 6 or 8 . A member of the FINISH study group will carry out randomisation when the patient is at the OR at the beginning of the operation. The randomisation will occur just prior to skin incision. The randomisation is a built-in property in the online eCRF system used in the trial (provided by Granitics, Espoo, Finland).

\section{Intervention}

Irrigation group

A burr-hole craniostomy is performed as described earlier. The dura is opened sharply and $10 \mathrm{~mL}$ of subdural exudate is aspired with blunt aspiration needle for a $\mathrm{CSDH}$ sample to be stored at $-75^{\circ} \mathrm{C}$ to be used for later analysis. Subdural space is irrigated by repeated rinsing with body temperature saline solution with a syringe and blunt needle until surgeon considers exudate to be clear. Minimum volume of irrigation will be $200 \mathrm{~mL}$ per operated side. The subdural drain is inserted $3-5 \mathrm{~cm}$ underneath the skull and parallel to it. Thereafter, operation is completed as described earlier. The total volume of irrigation as well as the duration of operation is recorded.

\section{No-irrigation group}

A burr-hole craniostomy is performed as described earlier. A small incision in the dura is made and $10 \mathrm{~mL}$ of subdural exudate is aspired with a blunt aspiration needle for a CSDH sample to be stored at $-75^{\circ} \mathrm{C}$ to be used for later analysis. The subdural drain is inserted approximately $3-5 \mathrm{~cm}$ underneath the skull and parallel to it. Thereafter, the operation is completed as described earlier. The duration of the operation is recorded.

\section{Blinding}

Due to the nature of the treatment, it is not possible to blind the surgeon and OR staff from the treatment allocation. Measures to minimise bias include:

- The randomisation is timed as closely as possible to the time of surgery (just prior to skin incision).

- The patient will not be informed of treatment allocation.

- Treatment allocation will not be documented in medical records (ie, all personnel participating in patient care after the operation will be blinded to allocation).

- The study group members collecting postoperative data, outcome data, imaging data and performing the statistical analyses will be blinded to treatment arm over the entire course of the trial, until the data analyses are carried out.

- The primary and secondary outcome measures are all evaluated in blinded matter, that is, the outcome assessor will be blinded with regard to treatment allocation

Emergency unblinding will occur only in exceptional circumstances when requested by the patient's clinical team (eg, need to treat a serious $\mathrm{AE}$ (SAE)), when knowledge of the actual treatment is essential for further management of the patient.

\section{Compliance to treatment allocation and possible crossover}

The per-protocol (PP) treatment is $0 \mathrm{~mL}$ of intracranial irrigation in the N-IR group and $\geq 200 \mathrm{~mL}$ (per operated side) of intracranial irrigation in the IR group. In the 
event of protocol breach, crossovers will be handled as follows:

- If the patient is randomised to the IR group and the intracranial irrigation volume is between $1 \mathrm{~mL}$ and $200 \mathrm{~mL}$, the patient is not considered a crossover.

- If the patient is randomised to the IR group and the intracranial irrigation volume is $0 \mathrm{~mL}$, the patient is considered a crossover (belongs to the N-IR group).

- If the patient is randomised to the N-IR group and 1-199 $\mathrm{mL}$ of intracranial irrigation is used, the patient is not considered a crossover.

- If the patient is randomised to the N-IR group and $\geq 200 \mathrm{~mL}$ of intracranial irrigation is used, the patient is considered a crossover (belongs to the IR group).

- In case of intervention failure (eg, not being able to insert subdural drain, intended or unintended drain removal before 36 hours), the patient is not considered a crossover.

\section{Primary outcome measure}

Our primary outcome measure is the rate of reoperations of ipsilateral CSDHs within 6 months.

\section{Indication for reoperation and reoperation technique}

The decision to proceed to reoperation is made by the treating neurosurgeon and will be made by the same indications as the primary operation (ie, symptom recurrence or insufficient resolution of clinical symptoms correlating to imaging findings (CT or MR imaging) of $\mathrm{CSDH}$ ). All reoperations will be conducted according to the current standard (ie, burr-hole with irrigation and subdural drain placement). In case of recurrence requiring reoperation, unblinding will not occur automatically, only in cases when the neurosurgery team treating the patient considers this information necessary for optimal care of the patient.

\section{Secondary outcome measures}

The study is not powered for secondary outcome measure comparisons and these outcomes (analyses) will be considered exploratory. The secondary outcomes include:

1. Modified Rankin Scale at 6 months after operation.

2. Mortality within 6 months of operation.

3. Duration of the operation.

4. Hospital length of stay (index hospital and need for further care).

5. CSDH volume reduction at 2 months after operation.

\section{Safety endpoints}

Safety endpoints within 6 months of operation, including the number and severity of AEs and procedure-related AEs (PRAE). AEs are categorised as SAEs and minor AEs (MAE). Procedure-related (severe and minor) AEs will be reported separately.

SAEs are defined as any inappropriate medical occurrence or effect that results in death, is life-threatening, requires hospitalisation or prolongation of an existing inpatient hospitalisation, results in persistent or significant disability or incapacity, or is another important medical event.

- Life-threatening in the definition of SAE refers to an event when the patient was at risk of death at the time of the event and does not refer to an event where the event might have hypothetically caused death. Prolonged hospitalisation due to delayed transfer will not be considered an AE or SAE.

Examples of SAEs are death, acute myocardial infarction, pulmonary embolism, systemic infection, acute cerebral infarction (PRAE), intracranial infection (PRAE), epileptic seizures (PRAE) and acute postoperative intracranial haematoma (PRAE).

MAEs are defined as clinically mild manifestations, referent to that the patient might be aware of the event or symptom but the event or symptom is easily tolerated by the patient.

- Examples of MAEs are local wound infection manageable with oral antibiotics (PRAE), abnormal skin bleeding from the wound (PRAE), other local infection manageable with oral antibiotics and deep venous thrombosis not causing pulmonary embolism.

\section{Follow-up}

The follow-up period is 6 months. We will arrange a clinical outpatient follow-up visit for all patients at 4-8 weeks postoperatively ( 6 weeks \pm 2 weeks). Before that, a postoperative brain CT will be performed. If the patient was preoperatively using any form of antithrombotic medication, the medication is not routinely restarted without reasonable clinical indication before the control brain CT. All recurrences requiring surgery within 6 months and complications within 6 months will be recorded. At 6 months, functional outcome (modified Rankin Scale (mRS) ) will be assessed by a FINISH study group member by phone interview. Further, for each patient, mortality will be verified through the Finnish Official Cause-ofDeath Statistics at 6 months. This statutory register is virtually $100 \%$ complete because each death, its associated official death-certificate, and the corresponding person information in the Finnish computerised population register are cross-checked.

\section{Sample size}

The trial is designed to ascertain whether drain without irrigation is non-inferior to drain with irrigation, with the rate of reoperations of ipsilateral CSDHs within 6 months as the primary outcome. We based the standard rate of reoperations $(9.6 \%)$ on the results from a recent Cochrane review that reported the recurrence rates after $\mathrm{CSDH}$ evacuation followed by subdural drainage in six randomised controlled trials (RCTs) with more than 30 patients per treatment arm. ${ }^{2}$ This yielded a maximum allowed margin of $9.0 \%$ to achieve non-inferiority. Following a consensus meeting with the trial investigators, the non-inferiority margin was lowered to $7.5 \%$. Thus, with a non-inferiority margin of $7.5 \%$, a $2.5 \%$ level of statistical significance (alpha $=0.025$ ) and an $80 \%$ power 
(beta $=0.20$ ), we will need 243 patients per study group. ${ }^{20}$ Accounting for a drop-out rate of $10 \%$, required group size increases to 270 per study group. Accordingly, we set the recruitment target at 540 patients.

\section{Data management}

All study data will be stored in an eCRF provided by Granitics (Espoo, Finland). Data are entered locally by the local research team. On receipt of the data, the FINISH personnel, blinded to the group allocation, will make a visual check of the data and query all missing, implausible and inconsistent data. Hospital patient records will also be used to collect missing data and to interpret inconsistent or implausible data. Participant files will be maintained in storage (both in electronic and paper format) at the coordinating centre for a period of 15 years after completion of the study.

\section{Data sharing}

Data generated by our study will be made available as soon as possible and will be available on reasonable request. Data access requests will be reviewed by the FINISH steering group. Requestors will be required to sign a data access agreement. Only anonymised data will be shared.

\section{Statistical analysis}

The statistical analysis will be performed both according to intention-to-treat (ITT) and PP principles. We will claim non-inferiority of single burr-hole evacuation without irrigation and subdural drainage only if this outcome is supported both by the ITT and the PP analysis. The ITT analysis will be performed using the full analysis set (FAS), defined as all randomised patients in the groups allocated to by the randomisation. No exclusions other than caused by missing information will be made. No imputation will take place. The PP analysis will be performed on the subset of the FAS that is compliant with the protocol, have a completed treatment, available measurements and no major protocol violations nor entry criteria violations.

Summary statistics will be presented for both groups. Continuous variables will be presented in terms of mean values or medians with SDs and IQRs, respectively. Categorical variables will be presented with relative frequencies in percent.

The results from the statistical analysis will be considered to support a claim of non-inferiority if the upper limit of a one-sided $97.5 \%$ CI (or equivalently a $95 \%$ twosided CI) excludes a difference in the primary endpoint in favour of the IR of more than $7.5 \%$. The centre stratification of the randomisation will be accounted for in the calculation of the CI.

Exploratory analyses of secondary and other binary endpoints will be performed using the $\mathrm{X}^{2}$ test or logistic regression analysis. Continuous outcomes will be analysed using Student's t-test or analysis of covariance. Potential effect modifiers (patient age, unilateral vs bilateral $\mathrm{CSDH}$, use of antithrombotic medication, preoperative
$\mathrm{mRS}$ and preoperative clinical status, haematoma density, haematoma size and presence of membranes on preoperative imaging) will be analysed by including interaction terms in statistical models.

The primary endpoint will be investigated as described above using a CI, which is equivalent to using a noninferiority test with a one-sided $p$ value of 0.025 (or a two sided of 0.05). The statistical testing of other endpoints will also be performed using a two-sided significance level of 0.05 . The statistical analysis will be performed using appropriate statistical software packages.

Prior to the statistical analysis, a statistical analysis plan will be finalised and an independent statistician will approve a dataset with sufficient data quality for the statistical analysis. Another statistician blinded to treatment arm will perform the analyses.

\section{Blinded data interpretation}

As in previous studies, ${ }^{21}{ }^{22}$ we will interpret the results of the trial according to a blinded data interpretation scheme. ${ }^{23}$ In brief, an independent statistician will provide the Writing Committee of the FINISH trial with blinded results from the analyses with the groups labelled group A and group B. The Writing Committee will then contemplate the interpretation of the results until a consensus is reached and all alternative interpretations of the findings are agreed on in writing. Once a consensus is reached, we will record the minutes of this meeting in a document coined 'statement of interpretation', which will be signed by all members of the Writing Committee. Only after reaching this common agreement will the data manager and independent statistician break the randomisation code and the correct interpretation chosen. A manuscript will then be prepared and finalised for the publication of the results. Detailed minutes of blinded data interpretation meetings will be provided as a supplement to the trial manuscript.

\section{Patient and public involvement}

To achieve a more patient-friendly design for our trial, we recruited five patient experts from the European Patients' Academy on Therapeutic Innovation (EUPATI Finland, https://fi.eupati.eu/) while designing the study. They were asked to review the informed consent form and questionnaires of the study. Further, these experts were asked to assess the burden of the intervention, time required to participate in the study, and outcomes all of which they estimated to be reasonable. After the FINISH study is completed, we will deliberate together with EUPATI Finland on how to share the study results with the general public.

\section{Data safety and monitoring committee}

Study monitoring is provided by the Clinical Research Institute of Helsinki University Hospital, who will ensure the quality of data collection and trial integrity. The monitoring is performed in accordance with currently valid rules and regulations, Good Clinical Practice and 
the standardised instructions of the Clinical Research Institute Helsinki University Hospital.

The members of the data safety and monitoring committee (DSMC) are neurosurgeons independent of the trial and have neither financial nor scientific conflicts of interest with the trial. The DSMC will oversee the interim analyses. The purpose of the interim analysis is safety surveillance. The interim analyses are performed after 50, 100 and 200 patients. No efficacy-related early stopping is planned.

\section{Ethics and dissemination}

The study was approved by the institutional review board (IRB) of the Helsinki and Uusimaa Hospital District on 13 November 2019 (HUS/3035/2019 §238, updated 26 February 2020).

All participating centres will obtain local institutional research approvals for the consent form template, the eCRF and any additional protocol amendments. Any protocol amendment will be communicated to the site investigators, the IRB, trial participants and trial registries as necessary.

Information about the study participants will be kept confidential and will be managed in accordance with the following rules: (1) all study-related information is stored securely at the clinical sites, (2) all possible study participant information in paper form is stored in locked file cabinets and is accessible only to study personnel, (3) all CRFs are identified only by a coded patient number, (4) all records that contain patient names or other identifying information are stored separately from the study records that are identified only by the coded patient number and (5) all local databases are password protected.

The results of the study will be published in an international journal and presented at (inter)national congresses. Trial results will be disseminated to the public in collaboration with EUPATI Finland.

\section{DISCUSSION}

To the best of our knowledge, this is the first large scale multicentre RCT comparing intraoperative irrigation with no intraoperative irrigation after burr-hole craniostomy and subdural drain placement for CSDH. The incidence of CSDH in Finland is approximately 18/100 000 , reaching as high as 130/100 000 in persons over 80 years old. ${ }^{24}$ As a consequence of the ageing population, more frequent use of antithrombotic medication and the improved access to diagnostics in most high-income countries, the incidence of $\mathrm{CSDH}$ is expected to increase in the future. ${ }^{25}$ The risk of complications following CSDH is rather low, but reducing the risk of recurrence is essential to avoid over-hospitalisation of otherwise fragile patients, which could be detrimental. ${ }^{26}$ Current studies examining strategies to decrease risk of recurrence include the Swedish study of irrigation-fluid temperature in the evacuation of CSDH (SIC!), ${ }^{27}$ the Dutch dexamethasone therapy versus surgery for CSDH (DECSA trial), ${ }^{28}$ the British dexamethasone for adult patients with a symptomatic CSDH trial, ${ }^{29}$ and two Canadian studies looking at the role of tranexamic acid in the treatment of CSDHs (TRACS trial, NCT02568124 $4^{30}$ and TRACE trial, NCT03280212).

A multicentre RCT that could show a decrease in recurrence rates has the potential to set a new gold standard of therapy, which would influence the treatment of these patients all over the world. If subdural irrigation fails to show any benefit over no irrigation, it would translate to a reduction in the risk of iatrogenic surgical complications and shortened operation times. It may also enable opportunities to develop newer, minimally invasive surgical techniques, including only subdural drain placement. This would not only benefit the individual patient but also healthcare systems all over the world, considering the sharply increasing incidence of CSDH.

A major strength of the study is that the five participating centres cover $100 \%$ of the Finnish population in terms of provision of neurosurgical care. In Finland, the surgical treatment of CSDH is exclusively carried out in University Hospital clinics, meaning that the follow-up regarding the primary endpoint (recurrence) should be $100 \%$. Also, in a highly digitalised healthcare system (local electronic healthcare databases since the early 2000s and nationwide electronic healthcare database since 2010) where every citizen has a unique personal identification number, the chances for successful follow-up regarding other endpoints is extremely high. A limitation is that it is impossible to blind the treating surgeon in relation to the treatment arm (irrigation or no irrigation). Furthermore, we cannot adjust for subtle differences in surgical technique between surgeons, although all participating centres as a whole perform the surgeries similarly. For example, the normal surgical technique involves irrigation until the fluid is deemed to be clear. However, in order to ensure a sufficient amount irrigation, we set a minimum threshold of $200 \mathrm{~mL}$ (per side).

\section{TRIAL STATUS}

The trial started recruiting patients in January 2020 in Helsinki and the other centres will start recruiting during the spring of 2020.

\section{Study administration structure}

Writing Committee: Pihla Tommiska, Rahul Raj, Christoph Schwartz, Teemu Luostarinen, Jarno Satopää, Simo Taimela, Teppo Järvinen, Jussi Posti, Teemu Luoto, Ville Leinonen, Sami Tetri, Timo Koivisto and Kimmo Lönnrot.

Steering Committee: Kimmo Lönnrot (chair), Riku Kivisaari (cochair), Teemu Luostarinen (cochair), Rahul Raj, Jussi Posti, Teemu Luoto, Ville Leinonen, Sami Tetri and Timo Koivisto.

Methods Centre: Helsinki University Hospital: Rahul Raj (principal investigator), Jarno Satopää (coprincipal 
investigator), Pihla Tommiska (coprincipal investigator, data management), Maarit Tuomisto (research coordinator).

Central Adjudication Committee: Kimmo Lönnrot, Riku Kivisaari, Teemu Luostarinen, Rahul Raj, Jussi Posti, Teemu Luoto, Ville Leinonen, Sami Tetri and Timo Koivisto.

Data Safety Monitoring Board: Martin Lehecka (MD, $\mathrm{PhD}$, A. Prof, Helsinki University Hospital, Finland), Frederick A. Zeiler (MD, PhD, A. Prof, Rady Faculty of Health Sciences, University of Manitoba, Canada), Jiri Bartek Jr. (MD, Karolinska University Hospital, Stockholm, Sweden).

\section{Author affiliations}

${ }^{1}$ Department of Neurosurgery, University of Helsinki and Helsinki University Hospital, Helsinki, Uusimaa, Finland

${ }^{2}$ Department of Neurosurgery, University Hospital Salzburg, Paracelsus Medical University, Salzburg, Austria

${ }^{3}$ Division of Anaesthesiology, Department of Anaesthesiology, Intensive Care and Pain Medicine, University of Helsinki and Helsinki University Hospital, Helsinki, Uusimaa, Finland

${ }^{4}$ Finland and Finnish Centre for Evidence-Based Orthopedics (FICEBO), University of Helsinki, Helsinki, Finland

${ }^{5}$ Department of Orthopaedics and Traumatology, University of Helsinki and Helsinki University Hospital, Helsinki, Uusimaa, Finland

${ }^{6}$ Clinical Sciences, Lunds Universitet, Lund, Sweden

${ }^{7}$ Division of Clinical Neurosciences, Department of Neurosurgery and Turku Brain Centre, Turku University Hospital and University of Turku, Turku, Finland

${ }^{8}$ Department of Neurosurgery, Tampere University Hospital and Tampere University, Tampere, Finland

${ }^{9}$ Department of Neurosurgery, Kuopio University Hospital and University of Eastern Finland, Kuopio, Pohjois-Savo, Finland

${ }^{10}$ Unit of Clinical Neuroscience, Neurosurgery, University of Oulu and Medical Research Center, Oulu, Finland

\section{Twitter Rahul Raj @rahulbrraj and Jonas Ranstam @jonasranstam}

Acknowledgements We wish to thank the five EUPATI patient experts participating in the design of the study. Pihla Tommiska has received personal research grants from The Finnish Medical Foundation, Orion Research Foundation sr and Maire Taponen Foundation. Jussi Posti is funded by the Academy of Finland (grant \#17379) and the Maire Taponen Foundation.

Contributors KL, RR, CS, RK, JS, TML, TJ and ST designed the trial. PT, RR, CS, TML, JS, ST, TJ, JF, JR, JP, TML, VL, ST, TK and KL have been involved in drafting the manuscript or revising it critically for important intellectual content. All authors read and approved the final manuscript.

Funding This study was supported by the State funding for university-level health research (Helsinki University Hospitals), Finska Läkaresällskapet, Medicinska Understödsföreningen Liv \& Hälsa.

Disclaimer The funding source will have no role in the collection, analysis and interpretation of data; in the writing of the report and in the decision to submit the article for publication.

Competing interests None declared.

Patient and public involvement Patients and/or the public were involved in the design, or conduct, or reporting, or dissemination plans of this research. Refer to the Methods section for further details.

Patient consent for publication Not required.

Provenance and peer review Not commissioned; externally peer reviewed.

Open access This is an open access article distributed in accordance with the Creative Commons Attribution Non Commercial (CC BY-NC 4.0) license, which permits others to distribute, remix, adapt, build upon this work non-commercially, and license their derivative works on different terms, provided the original work is properly cited, appropriate credit is given, any changes made indicated, and the use is non-commercial. See: http://creativecommons.org/licenses/by-nc/4.0/.
ORCID iDs

Rahul Raj http://orcid.org/0000-0003-4243-9591

Simo Taimela http://orcid.org/0000-0001-6755-2983

Teppo Järvinen http://orcid.org/0000-0003-3713-956X

Kimmo Lönnrot http://orcid.org/0000-0003-4361-1007

\section{REFERENCES}

1 Kolias AG, Chari A, Santarius T, et al. Chronic subdural haematoma: modern management and emerging therapies. Nat Rev Neurol 2014;10:570-8.

2 Peng D, Zhu Y. External drains versus no drains after burr-hole evacuation for the treatment of chronic subdural haematoma in adults. Cochrane Database Syst Rev 2016:CD011402.

3 Gurelik M, Aslan A, Gurelik B, et al. A safe and effective method for treatment of chronic subdural haematoma. Can J Neurol Sci 2007;34:84-7.

4 Lee C, Park DS, Song SW. Effect of intra-operative saline irrigation during Burr hole surgery on the recurrence for chronic subdural hematomas. The Nerve [Internet]. Korean Society of peripheral nervous system 2015;1:26-9.

5 Jang K-M, Kwon J-T, Hwang S-N, et al. Comparison of the outcomes and recurrence with three surgical techniques for chronic subdural hematoma: single, double Burr hole, and double Burr hole drainage with irrigation. Korean J Neurotrauma 2015;11:75-80.

6 Iftikhar M, Siddiqui UT, Rauf MY, et al. Comparison of irrigation versus no irrigation during Burr hole evacuation of chronic subdural hematoma. J Neurol Surg A Cent Eur Neurosurg 2016;77:416-21.

7 Ishibashi A, Yokokura Y, Adachi H. A comparative study of treatments for chronic subdural hematoma: Burr hole drainage versus Burr hole drainage with irrigation. Kurume Med J 2011;58:35-9.

8 Suzuki K, Sugita K, Akai T, et al. Treatment of chronic subdural hematoma by closed-system drainage without irrigation. Surg Neurol 1998;50:231-4.

9 Wang Q-P, Yuan Y, Guan J-W, et al. A comparative study of irrigation versus no irrigation during Burr hole craniostomy to treat chronic subdural hematoma. BMC Surg 2017;17:99.

10 Zakaraia AM, Adnan JS, Haspani MSM, et al. Outcome of 2 different types of operative techniques practiced for chronic subdural hematoma in Malaysia: an analysis. Surg Neurol; 2008;69:608-15 https://www.sciencedirect.com/science/article/pii/ S0090301907001978?via\%3Dihub

$11 \mathrm{Kim} \mathrm{DH}$, Kim HS, Choi HJ, et al. Recurrence of the chronic subdural hematoma after Burr-Hole drainage with or without intraoperative saline irrigation. Korean J Neurotrauma 2014;10:101-5.

12 Kuroki T, Katsume M, Harada N, et al. Strict closed-system drainage for treating chronic subdural haematoma. Acta Neurochir 2001:143:1041-4.

13 Ihab Z. Pneumocephalus after surgical evacuation of chronic subdural hematoma: is it a serious complication? Asian J Neurosurg 2012;7:66-74.

14 Seung W-B, Jeong JH. Postoperative subarachnoid hemorrhage and Multipunctate intracerebral hemorrhages following evacuation of bilateral chronic subdural hematomas. Korean $J$ Neurotrauma 2017;13:149-52.

15 Piaggio G, Elbourne DR, Pocock SJ, et al. Consort group for the. reporting of Noninferiority and equivalence randomized trials. JAMA 2012;308:2594

16 Chan A-W, Tetzlaff JM, Gøtzsche PC, et al. Spirit 2013 explanation and elaboration: guidance for protocols of clinical trials. BMJ 2013;346:e7586.

17 World Medical Association. World Medical association Declaration of Helsinki: ethical principles for medical research involving human subjects. JAMA 2013;310:2191-4.

18 Santarius T, Kirkpatrick PJ, Ganesan D, et al. Use of drains versus no drains after burr-hole evacuation of chronic subdural haematoma: a randomised controlled trial. Lancet 2009;374:1067-73.

19 Glancz LJ, Poon MTC, Coulter IC, et al. Does drain position and duration influence outcomes in patients undergoing Burr-Hole evacuation of chronic subdural hematoma? lessons from a UK multicenter prospective cohort study. Neurosurgery 2019;85:486-93.

20 Blackwelder WC. "Proving the null hypothesis" in clinical trials. Control Clin Trials 1982;3:345-53.

21 Sihvonen R, Paavola M, Malmivaara A, et al. Arthroscopic partial meniscectomy versus sham surgery for a degenerative meniscal tear. N Engl J Med 2013;369:2515-24.

22 Paavola M, Malmivaara A, Taimela S, et al. Subacromial decompression versus diagnostic arthroscopy for shoulder 
impingement: randomised, placebo surgery controlled clinical trial. BMJ 2018;362:k2860.

23 Järvinen TLN, Sihvonen R, Bhandari M, et al. Blinded interpretation of study results can feasibly and effectively diminish interpretation bias. J Clin Epidemiol 2014;67:769-72.

24 Rauhala M, Luoto TM, Huhtala $\mathrm{H}$, et al. The incidence of chronic subdural hematomas from 1990 to 2015 in a defined Finnish population. J Neurosurg 2019:1147-57.

25 Ducruet AF, Grobelny BT, Zacharia BE, et al. The surgical management of chronic subdural hematoma, 2010. Available: https:// link.springer.com/content/pdf/10.1007\%2Fs10143-011-0349-y.pdf [Accessed 28 Jun 2019].

26 Bartek J, Sjåvik K, Kristiansson H, et al. Predictors of recurrence and complications after chronic subdural hematoma surgery: a population-based study. World Neurosurg 2017;106:609-14.
27 Bartley A, Jakola AS, Bartek J, et al. The Swedish study of Irrigationfluid temperature in the evacuation of chronic subdural hematoma (SIC!): study protocol for a multicenter randomized controlled trial. Trials 2017:18:471

28 Miah IP, Holl DC, Peul WC, et al. Dexamethasone therapy versus surgery for chronic subdural haematoma (DECSA trial): study protocol for a randomised controlled trial. Trials 2018;19:575.

29 Kolias AG, Edlmann E, Thelin EP, et al. Dexamethasone for adult patients with a symptomatic chronic subdural haematoma (Dex$\mathrm{CSDH}$ ) trial: study protocol for a randomised controlled trial. Trials 2018:19:670

30 lorio-Morin C, Blanchard J, Richer M, et al. Tranexamic acid in chronic subdural hematomas (TRACS): study protocol for a randomized controlled trial. Trials 2016;17:235. 